\title{
Intelligent modeling and control of a conveyor belt grain dryer using a simplified type 2 neuro-fuzzy controller
}

\begin{abstract}
In this article, a nonlinear autoregressive with exogenous input (NARX) network was utilized to model a conveyor belt grain dryer using a set of inputï output data collected during an experiment to dry paddy grains. The resulting NARX model has achieved a remarkable modeling accuracy compared to other previously reported modeling techniques. To control the considered dryer, a simplified type 2 adaptive neuro-fuzzy inference system (ANFIS) controller was proposed. The effectiveness of this controller was demonstrated by several performance tests conducted by computer simulations. Moreover, a comparative study with other related controllers further confirmed the superiority of the proposed dryer controller.
\end{abstract}

Keyword: Conveyor belt grain dryer; Genetic algorithm; System identification; Type 2 neuro-fuzzy controller 